\title{
The Impact of Laryngeal pathology on sleep disorders patients
}

\author{
Grigore Raluca ${ }^{1,2}$, Condeescu Mihnea', Antonie-Simion Catrinel', Bejenaru Paula', \\ Munteanu Gloria', Cirstea Anca', Othman Amer', Rujan Simona', Taher Bianca', \\ Nitu Liliana ${ }^{1,2}$, Nicolaescu Alexandru', Bertesteanu Serban ${ }^{1,2}$ \\ 'Coltea Clinical Hospital ENT department (ROMANIA) \\ 2University of General Medicine and Pharmacy "Carol Davila" Bucharest (ROMANIA) \\ Mihnea.condeescu@gmail.com
}

\begin{abstract}
Sleep disorders are a common disorder the most frequent being obstructive sleep apnea characterized by repetitive episodes of upper airway collapse and respiratory pause. It is associated with a significant cardiovascular morbidity and mortality and severely alters the quality of life. In many cases it can remain undiagnosed because of its symptoms that can be underestimated by both the patient and the doctor. Tobacco, alcohol abuse and obesity are amongst the most common risk factors but the most important ones are related to different anatomical structures that can stop the airflow.Treating this disorder sometime requires multilevel surgery depending on the structures involved the most common of them being palatal, oropharyngeal and tongue base. The development and increased usage of sleep endoscopy has somewhat changed the treatment options due to the fact that laryngeal collapse is almost always seen only on sleep endoscopy. The role of laryngeal pathology has to be taken into account especially in cases where CPAP and surgery have failed .
\end{abstract}

Keywords: obstructive sleep apnoea; sleep endoscopy, larynx

\section{Introduction}

Sleeping is basic recurring process characterized altered consciousness, reduced muscle activity and reduced interactions with the surroundings. It is an important process allowing the brain and body to recover from daily aggressions, thus making it necessary for good health and good running of the physiological processes of our body. Good sleep is not only measured in the number of hours slept but also in the quality of the sleep. This being said, sleep disorders are a common group of disorders that affect the quality of sleep and can have an important effect on the health of the patients. The consequences of untreated sleep disorders can lead to daytime fatigue and sleepiness affecting the patient's daily activities. In time sleep disorders can lead to cardio-vascular problems like hypertension and heart attacks, diabetes and strokes. Despite having potentially severe effects on the patients sleep disorders tend to be underdiagnosed due to the fact that sometimes their symptoms are easily ignored by the patients. Traditionally otorhinolaryngologists tend to concentrate on the most common sites for upper airway obstructions like the oropharynx or tongue base but recently more studies have shown that the larynx can play an important role in sleep disorders.

\section{Diagnosis}

Although sleep disorders are a large group, three entities are important from an ENT point of view 
and warrant a possible surgical option: snoring, upper airway resistance syndrome and obstructive sleep apnea. Symptoms usually are classified in sleep symptoms and awake symptoms this having an important role in the diagnosis because awake symptoms are usually easily ignored by the patient while sleep symptoms are usually unknown for the patients, thus when taking a patient history is important to ask the relatives (wife, girlfriend) about the sleep symptoms.

Sleep symptoms are usually snoring, choking or gasping for air, witnessed apneas, gastroesophageal reflux and nocturia. Daytime symptoms range from anxiety, depression, morning headaches to sexual dysfunction, mood and personality changes, neurocognitive impairment and excessive daytime sleepiness. Altough obstructive sleep apnea and upper airway respiratory syndrome share most of the symptoms there are some slight differences. While in obstructive sleep apnea men are more commonly affected than females and patient are usually obese in the upper respiratory syndrome there is no significant gender difference and patients are usually non-obese [1]. Also patients with UARS complain more about daytime fatique and insomnia while OSA patient complain about excessive daytime sleepiness.

The gold standard for diagnosis of sleep disorders is represented by polysomnography but this method of diagnosis is not useful in pointing the anatomical structure that is involved in sleep disorders. The development of drug induced sleep endoscopy or DISE is of great importance in diagnosing and treating sleep disorders as obstructive sleep apnea because it is the closest thing to observing in real time the way the anatomical structures are involved in sleep disorders and can pinpoint the site of obstruction during sleep

\section{Methods and Results}

We present some cases in which laryngeal pathologies have caused sleep disorders. Laryngeal pathologies like benign tumors or even malignant tumors usually have a slow growth rate and usually can go unnoticed for long period of times until important symptoms affect the patient wellbeing. As stated above sleep disorders symptoms are usually ignored by the patients. So even though the patients have sleeping problems due to the obstruction of the airway they tend to see a doctor when more important symptoms like dysphonia or dysphagia appear.

One of the common laryngeal pathologies that can have an impact on the quality of sleep is Reinke's edema or polypoid corditis. These are benign lesions consisting in the alteration of the lamina propria [2] that usually have a slow growth rate with progressive symptoms that usually are ignored by the patients. In some cases then disease can grow to such proportions that the airway can become compromised.
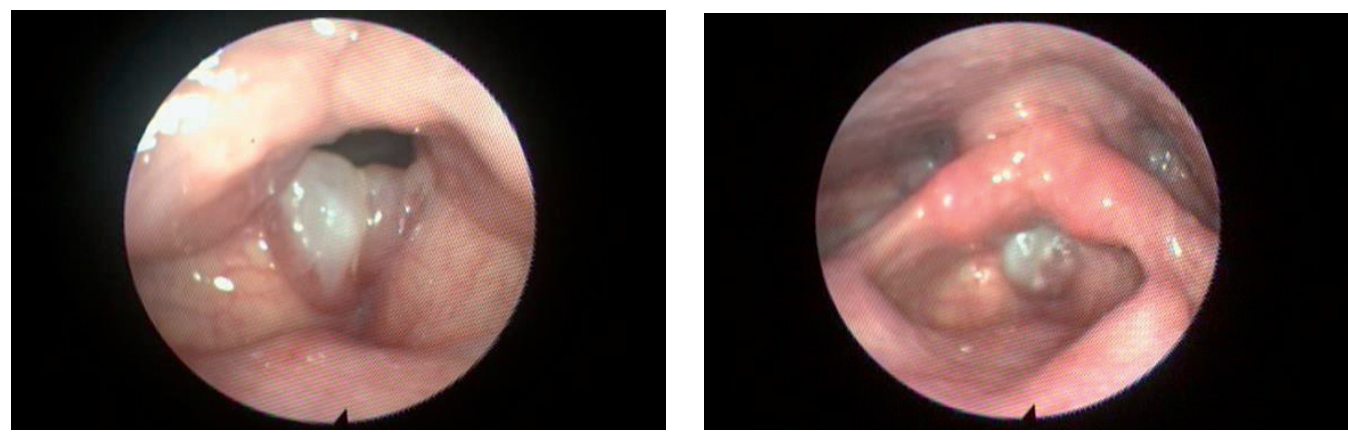

Fig. 1(a, b). The aspect of Reinke's edema on fibroscopy

We had 10 patients with Reinke's edema programmed for surgical intervention. Upon admittance in the ENT departmentpatients were also asked to complete the Epworth questionnaire. The mean score for the questionnaire was 14 before undergoing the surgical intervention. The mean score for Epworth at 3 months after surgery was 10. This improvement shows that at least subjective the patients felt better after treating the Reinke's edema.

Another possible laryngeal rare cause of sleep disorders is laryngocels. Laryngocels are abnormal dilatations of the laryngeal saccule filled with air. They can extend upward within la laryngeal ventricule and usually are in communication with the laryngeal lumen [3]. They are classified as internal 
laryngocel when contained within the false vocal cord or combined laryngocels when they protrude through the thyrohyoid membrane to the neck. They are slow growing and may be asymptomatic only being diagnosed in some cases by accidental imaging studies. Although many of them are asymptomatic when the grow in size they can affect the patient sleep by obstructing the airway.
We had one case of an combined laryngocel in a 45 year old female that upon admittance was asked to complete the Epworth Sleepiness Scale before undergoing surgery. The ESS scorebefore surgery was 18 . The patient was also a smoker and overweight.
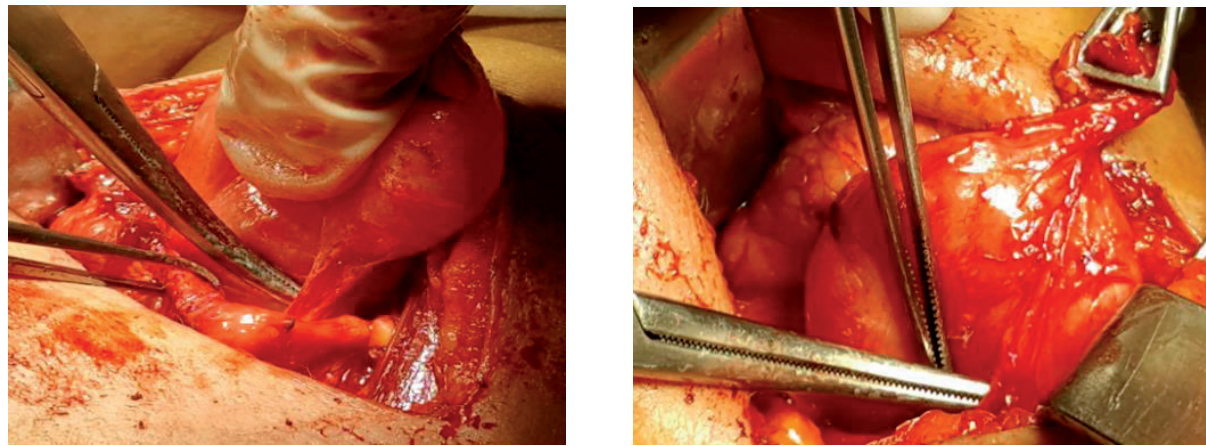

Fig. 2(a,b). Images from the surgery removing the combined laryngocel by external approach.

After surgery the patient ESS score was 12 showing that laryngocels can affect sleep quality and can have a role in sleep disorders.

In cases of more frequent benign laryngeal lesions like vocal cord polyps or epiglottic cysts their slow growth rate means that sometimes they can get to large sizes before the symptoms start troubling the patient. In 14 cases of vocal cord polyps and epiglottic cysts the mean ESS score was about 14 and after removal of the lesions the mean score was 11 which show improvement in the subjective daytime sleepiness in patients.

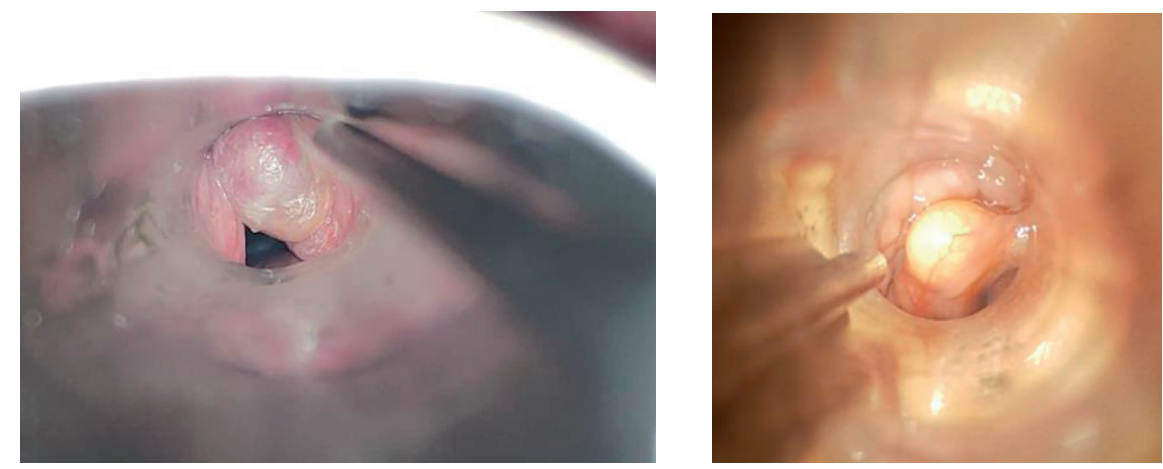

Fig. 3 (a,b). Vocal cord polyp. Epiglottic cyst

\section{Disscusion}

Altough the most frequent sites of obstruction in sleep disorders are located at palatal, oropharyngeal, and tongue base more and more studies have shown that the larynx can have an important role in sleep disorders. Hybášková et al [4] showed that pathology of the larynx (epiglottis) was observed in 16 patients $(31.4 \%)$ and that the use of drug induced endoscopy changed the surgical plan in $60.8 \%$ of the patients. Azarbarzin et al [5] showed that endoscopic studies reveal that epiglottic collapse renders patients at higher risk of failed oral appliance therapy or accentuated collapse on continuous positive airway pressure. Torre et al [6] have shown that epiglottic collapse in OSA range from $15 \%-70 \%$. These numbers mean that in some cases more aggresive surgical options are needed like epiglottopexy with/without lingual tonsillectomy, partial laser epiglottectomy,transcervical tongue base reduction with hyoepiglottoplasty. 
Altough epiglottic collapse is considered as an important factor in sleep disorders other laryngeal pathologies may interfere with normal sleep leading to a poor quality of life. Omer Araz et al [7] have shown that in some cases laryngocels and epiglottic cysts can cause sleep apnea. Vocal cord paralysis was also shown by Aziz L et al [8] to cause obstructive sleep apnea.

Hamdan et al [9] have shown that in some case Reinke's edema can cause or can be an important factor in sleep disorders especially obstructive sleep apnea. The slow growth and the fact that patients tend to ignore their symptomscan lead to compromised airway. Carefull endoscopic examination is needed in al patient with respiratory or sleep disorders.

\section{Conclussions}

The role of the larynx in sleep disorder and especially obstructive sleep apnea is not yet fully understood especially in cases of epiglottic collapse. Due to its anatomical position intuitively it was thought that the larynx can play an important role in sleep disorders and with therise in use of drug induced sleep endoscopy we are begin- ning to understand how the larynx may affect sleep. Further research is needed to understand the role the larynx plays in sleep disordersIn cases of lesions or tumours its almost certain that the airway is compromised in some degree and fast diagnosis and treatment can resolve the symptoms. This is important to know because a patient with sleep disorders has to be diagnosed and treated by a multidisciplinary team in which a complete ENT examination is warranted at all times.

\section{Conflict of Interest}

The authors have no conflict ofinterest.

\section{Conribution of Authors}

All authors have equally contributed to this work

\section{Ethical Approval}

All procedures performed in this study were in accordance with the ethical standard of the institution and with the 1964 Helsinki declaration and its later amendments.

\section{References}

1. Michael Friedman, Ofer Jacobowitz Sleep APnea And Snoring Surgical and nonsurgical therapy 2020 Elsevier Second edition.

2. Clark A Rosen, C Blake Simpson Operative Techniques in laryngology 2008 Springer

3. Karol Zelenik, Lucia Stanikova, Katarina Smatanova, Michal Cerny, and Pavel Kominek Treatment of Laryngoceles: What Is the Progress over the Last Two Decades?Endoscopic/External Approaches in Otorhinolaryngology and Head and Neck Surgery Volume 2014 Article ID 819453 https://doi.org/10.1155/2014/819453

4. Jaroslava Hybášková, Ondřej Jor, Vilém Novák, Karol Zeleník, Petr Matoušek, and Pavel Komínek, Drug-Induced Sleep Endoscopy Changes the Treatment Concept in Patients with Obstructive Sleep Apnoea, Volume 2016 Article ID 6583216 https://doi.org/10.1155/2016/6583216

5. Azarbarzin A, Marques M Sands SA, Op de Beeck S, Genta PR, Taranto-Montemurro L, de Melo CM, Messineo L, Vanderveken OM, White DP, Wellman A. Predicting epiglottic collapse in patients with obstructive sleep apnoea Eur Respir J. 2017 Sep 20; 50(3). pii: 1700345. doi: $10.1183 / 13993003.00345-2017$

6. Torre C, Camacho M, Liu SY, Huon LK, Capasso R Epiglottis collapse in adult obstructive sleep apnea: A systematic review Laryngoscope. 2016 Feb; 126(2): 515-23. doi: 10.1002/lary.25589

7. Araz, O., Turan, A., Yoruk, O. et al. Laryngocele and epiglottic cyst as rare causes of obstructive sleep apnea. Sleep Breath 13, 285-287 (2009). https://doi.org/10.1007/s11325-009-0250-x

8. Aziz L, Ejnell H. (2003) Obstructive sleep apnea caused by bilateral vocal fold paralysis Ear Nose Throat J. 2003 Apr; 82(4): 326-7.

9. Hamdan AL, Khalifee E, Abi Akl PR, Ghanem A, El Hage A, Pathogenic Role of Reinke's Edema in Snoring and Obstructive Sleep Apnea J Voice. 2018 Dec 15. pii: S0892-1997(18)30495-8. doi: 10.1016/j.jvoice.2018.12.001

10. Mario Milkov, Positional obstructive sleep apnea as a new interdisciplinary challenge. International Bulletin of Otorhinolaryngology, XV, 2019, 2: 16-20.

11. Mario Milkov. Trans-oral robotic surgery for obstructive sleep apnea - selection of patients. International Bulletin of Otorhinolaryngology, XV, 2019, 4: 38-45

12. Mario Milkov, Transoral Robotic Surgery for Treatment of Obstructive Sleep Apnea. International Bulletin of Otorhinolaryngology, XV, 2019, 4: $46-52$. 